\title{
Hill and Dale: A técnica de Edison e a transcrição de registros fonográficos de cilindros
}

\author{
Hill and Dale: The Edison technique and the transcription of phonographic records of cylinders
}

\author{
Nilson Souza Filho*1, J. Jeferson Rosa ${ }^{1}$, Daniel Matos Silva ${ }^{2}$, Nelson Guilherme Casteli Astrath ${ }^{2}$ \\ ${ }^{1}$ Engenharia Acústica, Centro de Tecnologia, Universidade Federal de Santa Maria, RS, Brazil \\ ${ }^{2}$ Departamento de Física, Universidade Estadual de Maringá, PR, Brazil
}

\begin{abstract}
Recibida en 26 de Setembro, 2017. Revisado en 05 de Novembro, 2017. Aceptado en 13 de Novembro , 2017.
Apresentamos uma revisão sobre a primeira técnica de gravação/reprodução de som e exemplos de transcrições dos principais tipos de suporte físico de gravação fabricados comercialmente entre 1889 e 1929 . As transcrições foram realizadas com um aparato que utiliza um mandril acoplado a um motor de passo, uma cápsula fonocaptora na extremidade de um braço giratório, uma interface de áudio e um computador, para transcrever o som registrado em cilindros em um formato digital. Os áudios foram identificados em catálogos e estão disponíveis na web.

Palavras-chave: Registro fonográfico, gravação mecânica; cilindro de cera, digitalização de som.
\end{abstract}

\begin{abstract}
We present a review of the first sound recording/reproduction technique and examples of transcriptions of the main types of recording media manufactured commercially between 1889 and 1929. The transcriptions were performed with an apparatus using a mandrel coupled to a stepper motor, a pickup phono cartridge at the end of a pivoting arm, an audio interface and a computer, to transcribe the sound recorded on the cylinders in a digital format. The audios were identified in catalogs and are available on the web.

Keywords: Phonographic record, mechanical recording, wax cylinder, sound digitization.
\end{abstract}

\section{Introdução}

Entre 1807 e 1845, Thomas Young, Wilhelm Weber e Jean-Marie Duhamel criaram dispositivos para registros gráficos de corpos vibrantes (vibrograph ou vibroscope) 1.2. Porém, eles não eram capazes de transcrever ondas acústicas. Essencialmente, tais dispositivos eram osciloscópios mecânicos.

Em 1857, Édouard-Léon Scott de Martinville inventou o Phonautograph, que era capaz de escrever ondas acústicas 2, 3]. O dispositivo era muito semelhante aos seus antecessores; a forma de onda era transcrita por uma ponta em contato com um cilindro rotativo [1,2]. O principal avanço do fonautógrafo foi a introdução de um sistema que envolvia um cone através do qual as ondas sonoras poderiam ser coletadas e direcionadas até uma membrana com uma pena assemblada para registrar o som em um papel com fuligem [2,3].

Assim como os vibroscópios, o fonautógrafo também não era capaz de reproduzir o som registrado. A reprodução de registros fonográficos teve início em 1877 , quando Edison inventou o Phonograph [1, 4].

O projeto original do fonógrafo (tinfoil) utilizava um sistema corneta+diafragma+agulha para gravar a onda acústica ao girar um cilindro coberto com uma folha de alumínio. Os sulcos gravados na folha de alumínio

*Endereço de correspondência: nilson.evilasio@eac.ufsm.br permitiram a reprodução do som gravado por um sistema recíproco agulha+diafragma+corneta [1, 4]. O conceito de reciprocidade, na transdução mecânica-acústica, estabelecido por Edison, ao utilizar uma folha de alumínio como um meio físico para gravar/reproduzir o som, é a semente da indústria fonográfica. O passo crucial para o surgimento de uma indústria voltada para comercialização de música foi o surgimento de um suporte físico de gravação com reciprocidade mais adequada.

O cilindro de cera [5, 6], foi o primeiro suporte físico de gravação comercial (media) e atingiu consumidores em larga escala de 1890 à 1910, sendo utilizado no Brasil para gravações etnográficas até $1937,7,8$. Os cilindros de cera e o fonógrafo de Edison foram utilizados por meio século, até perderem totalmente o espaço para os discos e o Gramophone de Berliner [9]. As empresas cessaram gradativamente as fabricações de fonógrafos com domínio comercial dos discos.

O Archéophone é uma versão moderna dos fonógrafos do início do século XX. Ele foi criado para poder ler todos os formatos de cilindros fonográficos de cera produzidos de 1889 à 1929 10]. Arqueofones têm sido utilizados para copiar e preservar registros fonográficos antigos em várias bibliotecas e museus ao redor do mundo [11], sendo, portanto, uma ferramenta científica importante para estudar as culturas e os modos de vida do passado a partir da análise de vestígios de som. 
As técnicas físicas de registro fonográfico podem ser classificadas em gravação mecânica, gravação magnética, e gravação óptica. As etapas de evolução da indústria fonográfica estão relacionadas ao suporte físico de gravação de cada técnica. Esta nota de aula apresenta uma breve revisão sobre os princípios da técnica de gravação mecânica Hill and Dale e os resultados de transcrições de músicas registradas em cilindros de cera, obtidos com um arqueofone de braço giratório.

\section{Técnicas de Gravação}

\subsection{Gravação Mecânica}

A técnica de gravação idealizada por Edison e utilizada nas gravações dos cilindros é conhecida como Hill and Dale, ou registro por corte vertical, para diferenciar da técnica de registro por corte lateral (ou horizontal) inventada por Berliner em 1887 [9] e utilizada em discos de goma-laca a partir de 1896 [12]. A figura 1 apresenta um esquema representativo das duas técnicas de gravação mecânica.

Desde que a energia do corte aplicado seja somente da pressão sonora transmitida pela corneta à ponta de corte através de um diafragma, em ambas as técnicas, a ondulação do sulco é diretamente proporcional às vibrações sonoras. No caso da técnica de registro por corte vertical, o som está relacionado com a profundidade dos sulcos.

\subsubsection{Corneta}

A corneta, em uma gravação, tem a função de captar o som e direcioná-lo ao diafragma, enquanto que na reprodução tem a função de amplificar o som do diafragma 13, 14. A figura 2 apresenta um fonógrafo de Edison com uma corneta típica.

Pelo teorema Reynolds, é possível afirmar que

$$
\frac{d m}{d t}=\frac{\partial}{\partial t} \int_{V} \rho d V+\int_{S} \rho(\vec{v} \cdot \hat{n}) d S=0,
$$

em que $m, \rho$ e $V$ são, respectivamente, a massa, densidade e o volume de gás na corneta, $S(y)$ é a área de integração ao longo do tubo, $\hat{n}$ é o vetor normal ao elemento de área
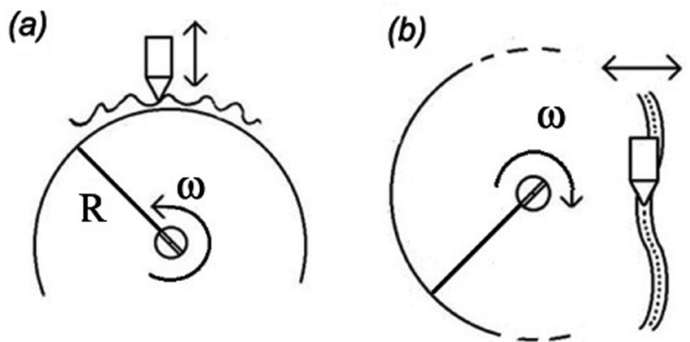

Figura 1: Técnicas de registro fonográfico por gravação mecânica: (a) Registro por corte vertical em cilindros (Edison, 1878) [4]; (b) Registro por corte lateral em discos (Berliner, 1896) [12].

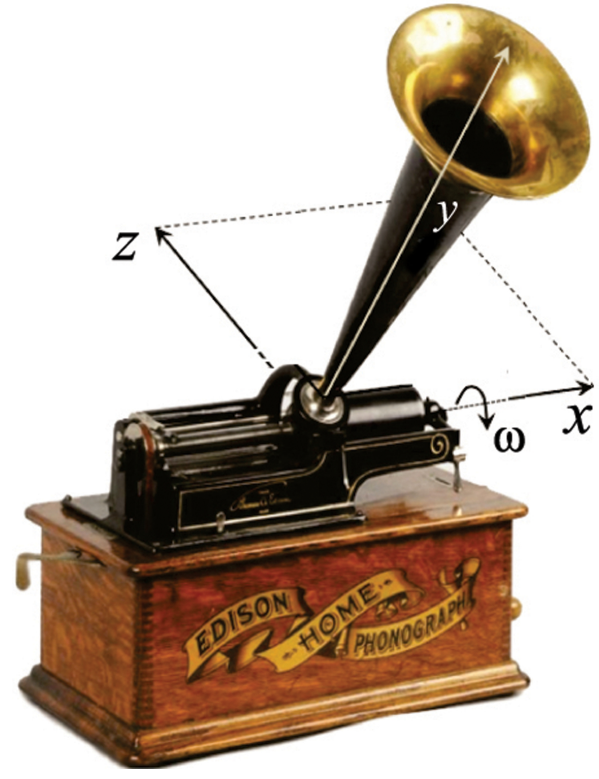

Figura 2: Fonógrafo de Edison com uma corneta típica, de comprimento $y=35,56 \mathrm{~cm}$ e raio $0,80<a<17,78 \mathrm{~cm}$ [14].

$d S$ e $\vec{v}$ é a velocidade do gás que atravessa a superfície de integração. Para obter um sistema fechado de equações, devemos assumir as seguintes condições:

- O fluido obedece a lei do gás ideal, $p=n k_{B} T$, em que $p$ é a pressão, $n$ o número de mols, $T$ a temperatura e $k_{B}$ a constante de Boltzmann;

- A forma do tubo é fixa e independente do tempo, mas não é constante em $y$, então pela equação 1 $\partial(\rho S) / \partial t=-\rho \partial S / \partial y$;

- A densidade de massa é constante em toda a área da seção transversal, mas depende do tempo;

- A segunda lei de Newton é sempre válida, logo $\rho(\partial v / \partial t)=\partial p / \partial y$

- O fluido é irrotacional, portanto, $\nabla \times \vec{v}=0$.

Ao resolver todas as equações para $p$ e supor que para tubos longos, a variação da pressão local é muito maior que a flutuação da densidade local, obtemos a equação de Webster

$$
\frac{1}{S} \frac{\partial}{\partial y}\left(S \frac{\partial p}{\partial y}\right)=\frac{1}{c^{2}} \frac{\partial^{2} p}{\partial t^{2}},
$$

em que $c=\sqrt{k_{B} T / m}$ é a velocidade do som e $y$ é a coordenada ao longo do tubo. A equação de Webster é uma aproximação unidimensional para ondas sonoras de baixa frequência ao longo de um tubo rígido com uma área de seção transversal variável $S(y)$. Pode ser pensada como uma equação de onda com um termo de origem que leva em consideração a geometria não linear do tubo 15, 16.

A trombeta de Gabriel (ou trompete de Torricelli) é uma aproximação do tipo de corneta mais comum encontrada nos fonógrafos de Edison [16]. Trata-se de uma superfície de revolução obtida ao girar uma hipérbole 
em torno de um eixo. É possível observar, pela corneta da figura 2 que

$$
r(y)=\frac{a}{y} \text { e } S(y)=\frac{\pi a^{2}}{y^{2}} .
$$

Então, a equação de Webster torna-se

$$
\frac{d^{2} p(y)}{d y^{2}}-\frac{2}{y} \frac{d p(y)}{d y}+\omega^{2} p(y)=0
$$

cuja solução é

$p(y)=\sqrt{2 / \pi \omega^{3}}[(A \omega y+B) \cos (\omega y)+(B \omega y-A) \sin (\omega y)]$

A potência acústica reunida por uma corneta de tamanho normal em um estúdio de gravação variava de $1 \mu W$ a $1 m W$ [17.

\subsubsection{Diafragma}

Um diafragma é um transdutor acústico-mecânico. É construído com uma membrana fina de bordas fixas. A membrana do diafragma é um disco plano de mica, vidro, alumínio ou cobre [18], que converte a pressão das ondas sonoras em vibrações mecânicas ao movimentar uma ponta que corta um sulco de som no suporte físico de gravação. A figura 3 apresenta uma foto de um dos vários modelos de diafragma de Edison.

Um diafragma modulado por ondas sonoras provenientes de uma corneta em uma gravação puramente mecânica, requer um movimento de velocidade constante para uma resposta plana. Ao assumir que o movimento do diafragma de gravação é uniforme e que ele não vibra em modos superiores, podemos desenvolver uma equação diferencial para o seu movimento [19]. Se $y(t)$ for o deslocamento da agulha e $v_{y}(t)$ a velocidade com que a agulha grava o sulco, para forças elásticas, temos

$$
F_{e}(t)=k \Delta y(t)
$$

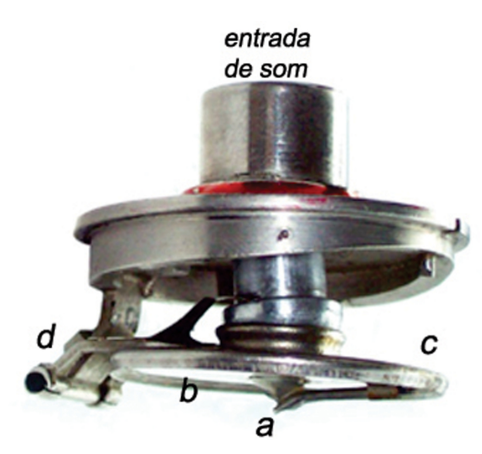

Figura 3: Foto de um diafragma de Edison, com um entrada circular de som de $1,60 \mathrm{~cm}$ de diâmetro. No ponto $\boldsymbol{b}$ há uma membrana circular de $3,30 \mathrm{~cm}$ de diâmetro e com bordas parafusadas a um aro $\boldsymbol{c}$. O ponto de gravação $\boldsymbol{a}$ é uma ponta de corte (safira) presa no centro da membrana e acoplada a uma alavanca $\boldsymbol{d}\left[\begin{array}{l|l}13 & 18\end{array}\right]$. sendo $k=(1 / C)$, a rigidez da membrana do diafragma com compliância $C$. Para forças viscosas

$$
F_{v}(t)=\mu v_{y}(t)
$$

em que $\mu$ é o coeficiente de atrito. Para forças inerciais

$$
F_{m}(t)=M \dot{v}_{y}
$$

em que Mé a massa da membrana do diafragma. Ao reunir todas as forças que atuam sobre o diafragma, temos

$$
F_{\text {total }}(t)=k \Delta y(t)+\mu v(t)+M \dot{v}_{y}=A p(t),
$$

em que $p(t)$ é a pressão do ar no final da corneta e $A$ é a área do diafragma. Ao derivar ambos os lados da equação 9. obtemos

$$
k v(t)+\mu \dot{v}_{y}+M \ddot{v}_{y}=A \dot{p} .
$$

Ao tomar a transformada de Laplace da equação 9 obtemos a função de transferência do deslocamento da membrana

$$
H(s)=\frac{Y(s)}{P(s)}=\frac{A}{\left(m s^{2}+\mu s+k\right)},
$$

enquanto que a transformada de Laplace da equação 10. $\operatorname{com} s=j \omega$, fornece a função de transferência da velocidade de gravação do diafragma

$$
H(s)=\frac{V(s)}{P(s)}=\frac{A}{k} \frac{j \omega}{\left[1-\left(\frac{\omega}{\omega_{0}}\right)^{2}+j \omega \frac{\mu}{k}\right]} .
$$

Para obter uma boa gravação ao longo das faixas de baixa e alta frequência, as seguintes condições devem ser obedecidas:

$$
\begin{gathered}
\frac{\mu}{k} \omega_{\min }>>1, \\
\frac{\omega_{\max }}{\omega_{0}}<<1,
\end{gathered}
$$

em que $\omega_{0}^{2}=k / M$ é a frequência de ressonância do gravador. A equação 13 determina que as forças viscosas devem predominar sobre as forças elásticas e a equação 14 que a frequência de ressonância esteja acima da maior frequência a ser registrada 19$]$.

\subsubsection{Agulha}

O cilindro é montado horizontalmente e girado de tal forma que o corte sobre a superfície da cera, se move da direita para a esquerda em relação ao observador (plano $x y)$. O sulco do cilindro é cortado com uma barra de safira cilíndrica, lapidada e polida para realizar cortes com um ângulo de 15 graus em relação ao eixo longitudinal $z$ e 10 graus em relação a normal da superfície da cera enquanto o cilindro gira no sentido horário com velocidade $\omega$ (plano $z y)$. 
O diafragma gravador é modulado perpendicularmente à superfície da cera e assume-se que a modulação é simétrica em relação à profundidade média do gravador. Portanto, um sulco não modulado terá de ser cortado à metade da profundidade máxima de modulação [17. Vamos considerar o sulco vertical cortado no cilindro, dado por

$$
y_{s}(t)=y_{0}+\Delta y \operatorname{sen}(\omega t),
$$

em que $y_{s}(t)$ é profundidade de corte, $y_{0}$ é a profundidade não modulada, $\Delta y$ é a profundidade de corte modulada pelo som que chega ao difragma e $\omega$ é a frequência angular do corte. A profundidade do sulco cortado deve ser a integral da onda de pressão que chega no fim do cone até o diafragma. Isso ocorre quando a velocidade do sinal cortado no cilindro é proporcional à pressão da onda sonora.

Se a faceta de corte produzir um sulco com profundidade de corte ligeiramente elíptica, podemos considerar que o sulco formado é aproximadamente um segmento circular. As barras de safira têm um diâmetro de $0,9 \mathrm{~mm}$ para gravações de dois minutos e $0,6 \mathrm{~mm}$ para gravações de quatro minutos 17]. A profundidade média dos sulcos na maioria das gravações profissionais era de aproximadamente $0,05 \mathrm{~mm} 17]$.

\subsubsection{Cilindros de cera}

As dimensões do cilindro de cera do tipo mais popular são de $55 \mathrm{~mm}$ de diâmetro e $105 \mathrm{~mm}$ de comprimento, com vazão cônica de diâmetro menor de $16 \mathrm{~mm}$ e diâmetro maior de $18 \mathrm{~mm}$ (figura 4). A trajetória do sulco no cilindro é dada pelo vetor posição

$$
\vec{r}(t)=\vec{x}(t) \hat{i}+\vec{y}(t) \hat{j}+\vec{z}(t) \hat{k},
$$

sendo

$$
\begin{gathered}
x(t)=v_{x} t, \\
y(t)=R \operatorname{sen}(\omega t), \\
z(t)=R \cos (\omega t),
\end{gathered}
$$

a gravação do sulco no cilindro, portanto, segue uma trajetória helicoidal em torno do raio $R=\left(D_{3} / 2\right)-y_{s}$. Aproximadamente 400 voltas de sulcos de som com passos de $254 \mu \mathrm{m}$, eram cortados sobre a superfície do cilindro [20]. Inicialmente eram 100 voltas por polegada, depois passou a ser 200tpi (threads per inch).

Desde a sua criação, o cilindro fonográfico passou por várias mudanças de material em sua constituição [20,21. Os cilindros de cera foram fabricados de duas maneiras para comercialização: cilindros virgens para serem gravados em fonógrafos com o diafragma para gravação e cilindros prensados já com os sulcos da gravação para serem tocados com o diafragma de reprodução [21].

Surgiram quatro tipos principais de cilindros, identificados de acordo com as cores: marrom; preto e azul. O cilindro de cera marrom foi o primeiro formato padrão

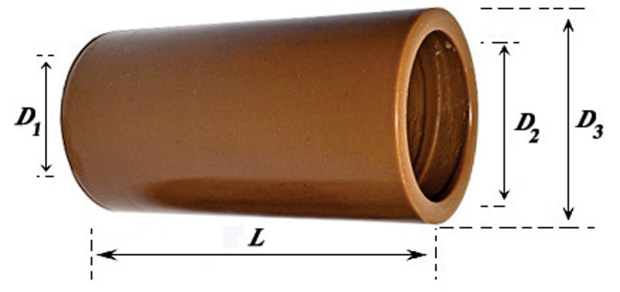

Figura 4: Dimensões do cilindro: diâmetro interno menor $D_{1} \approx$ $1,60 \mathrm{~cm}$; diâmetro interno maior $D_{2} \approx 1,80 \mathrm{~cm}$; diâmetro externo $D_{3} \approx 5,50 \mathrm{~cm}$ e comprimento $L \approx 10,50 \mathrm{~cm}(9,00 \mathrm{~cm}$ para gravação). A extremidade de diâmetro interno maior é a referência do início do registro.

de comercialização de música a partir de 1889, sua capacidade de armazenamento é de 2 minutos [17].

Os cilindros pretos, moldados com gravações, foram desenvolvidos por Edison, em 1902. O método consiste de um processo de eletrodeposição de ouro em um cilindro gravado e extraído um molde matriz para duplicação. Esse molde é oco com a impressão de um cilindro original no interior onde é colocada uma mistura de ceras fundidas 21,22]. Os cilindros mais populares moldados por este processo foram os Edison Gold-Moulded Record e Columbia Record, importados para o Brasil com gravações de músicas de até quatro minutos.

Em 1912 surgiu o cilindro azul com uma camada superficial de celulóide plástico, com capacidade de 4 minutos e 45 segundos. Os cilindros Edison Blue Amberol Recorded foram fabricados até 1929 .

Dentre os cilindros gravados no Brasil, predominavam os cilindros Phrynis e os cilindros raspados para regravação, depois novos materiais e formatos foram desenvolvidos, dentre eles os indestrutíveis, fabricados em celulose e outros materiais, os de longa duração (4 a 6 minutos) e os coloridos 20 .

A comercialização de cilindros virgens e cilindros raspados, possibilitou gravação imediata para fins domésticos e em campo. Essas características fazem com que o cilindro fonográfico também figure como media para outras finalidades [21], assim como aconteceu, posteriormente, com os discos em acetato com base em alumínio, com os meios magnéticos e com os meios digitais (fitas cassete, DAT e MD).

\section{Técnicas de leitura}

A alternativa mais simples para contornar os problemas de danos em cilindros fonográficos durante a reprodução com um diafragma original, é a utilização de cápsulas fonocaptoras. Para leitura de cilindros raros com deterioração avançada, trincados ou quebrados, é recomendado técnicas ópticas de leitura 23.24. 


\subsection{Cápsula fonocaptora}

A cápsula fonocaptora é um transdutor mecânico-elétrico que utiliza o conceito de estereofonia (som binaural) para ler discos de vinil que possuem sulcos em espiral registrados por corte lateral. Neste sistema, o corte e movimento do sulco tem ângulo de 45 graus, e cada parede é um canal (direito e esquerdo) 25]. A grande vantagem é a compatibilidade de leitura com o monofônico, registrados por corte vertical.

Existem vários tipos de cápsulas. As cápsulas magnéticas, por exemplo, surgiram em 1950 para substituir as cápsulas piezoelétricas. O funcionamento de uma cápsula magnética é com uma bobina que se move próximo à um imã fixo (moving coil) ou é com um imã que se move próximo à bobinas fixas (moving magnet).

A figura 5 apresenta uma cápsula fonocaptora magnetodinâmica (moving magnet). As agulhas das cápsulas fonocaptoras são de um material duro como a safira ou diamante e recebem um tratamento para que sua superfície fique extremamente lisa, de forma cônica, esférica ou elíptica. A agulha é presa a um cantiléver, uma haste metálica suspensa por um elastômero.

Este sistema pode ser considerado como um absorvedor de vibrações dinâmicas [26], pois consiste de uma massa auxiliar acoplada por uma mola à massa principal e a um elemento amortecedor.

O sistema de vibração, representado na figura 6, é descrito pelas equações diferenciais

$$
\begin{gathered}
m_{1} \ddot{y}_{1}+R\left(\dot{y}_{1}-\dot{y}_{2}\right)+k_{1} y_{1}+k_{2}\left(y_{1}-y_{2}\right)=A \sin (\omega t), \\
m_{2} \ddot{y}_{2}+R\left(\dot{y}_{2}-\dot{y}_{1}\right)+k_{2}\left(y_{2}-y_{1}\right)=0
\end{gathered}
$$

em que $m_{1}$ é a massa do ímã; $m_{2}$ é a massa da agulha, $R$ é o amortercimento do sistema absorvedor, $k_{1}$ é a rigidez do sistema principal de vibração, $k_{2}$ é a rigidez

(a)

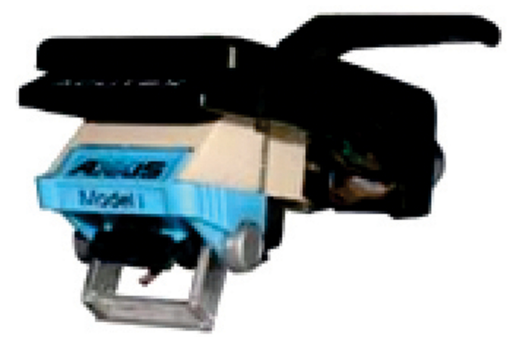

(b)

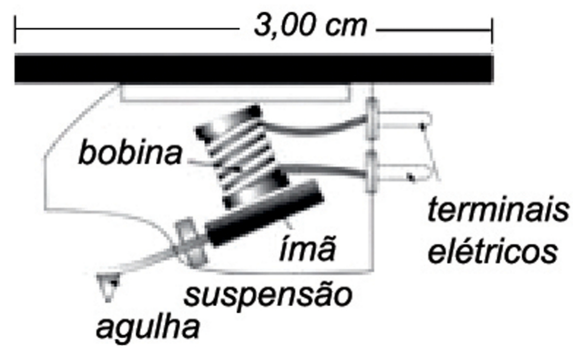

Figura 5: (a) Foto de uma cápsula fonocaptora; (b) Vista lateral do esquema de uma cápsula magnetodinâmica. (a)

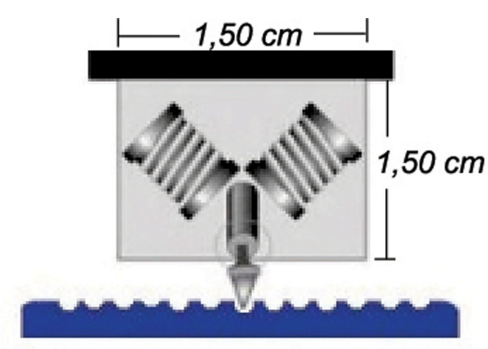

(b)

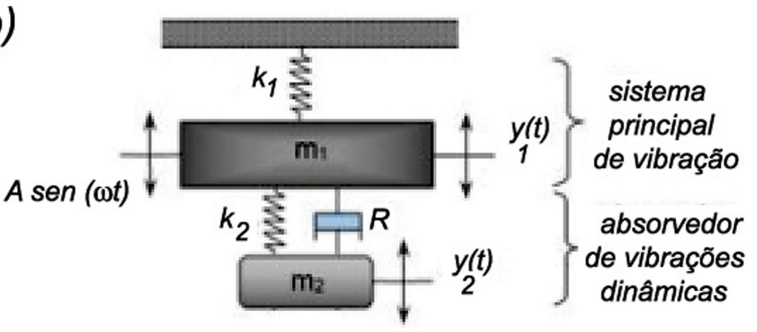

Figura 6: (a) Vista frontal do esquema da cápsula com a agulha sobre o sulco; (b) Sistema simples de vibração com absorvedor dinâmico 26.

do sistema absorvedor, $y_{1}$ é o deslocamento do ímã e $y_{2}$ é o deslocamento da agulha.

Em frequências bem abaixo da ressonância do sistema massa-mola absorvedor, as massas se movem em fase e não ocorre amortecimento. Acima da sua frequência de ressonância, a massa do absorvedor fornece um ponto fixo aparente no espaço, o que resulta em uma força no elemento de amortecimento que é transmitida ao sistema principal de vibração [26].

Quando o amortecedor inercial é sintonizado para vibrar logo abaixo da frequência de ressonância do sistema principal, a massa do absorvedor se desloca fora de fase com a massa principal na ressonância e amplifica a força de amortecimento [26]. O ajuste da agulha é dado por

$$
f_{a}=\frac{1}{1+m_{2} / m_{1}} f_{s}
$$

em que $f_{a}=(1 / 2 \pi)\left(k_{2} / m_{2}\right)^{1 / 2}$, é frequência de ressonância do absorvedor e $f_{s}=(1 / 2 \pi)\left(k_{1} / m_{1}\right)^{1 / 2}$, é a frequência de ressonância do sistema principal de vibração. O ajuste proporciona amplitudes iguais em duas frequências [26]. Este mecanismo permite a transdução mecânica-elétrica a partir das vibrações captadas pela agulha ao percorrer o sulco do cilindro. As vibrações sentidas pela agulha são transmitidas até o ímã. A variação do campo magnético nas bobinas ocasionado pela vibração do ímã, gera uma corrente elétrica que origina o sinal de áudio, proporcional ao som registrado no cilindro.

\section{Materiais e métodos}

\subsection{Cilindros para teste}

Foram adquiridos quatro tipos de cilindros fonográficos do selo Edison Records. 
Edison Record (Brow Wax Cylinder, 1889-1902)

Os cilindros de cor marrom tem a seguinte composição química: ácido esteárico $48,0 \%$; estearato de sódio $20,2 \%$; estearato de alumínio $11,3 \%$ e ceresina $20,5 \%$ [22]. Os primeiros cilindros de cera não têm informações impressas ou escritas sobre eles, por isso, apresentações faladas eram gravadas no início da maioria dos cilindros, uma prática que continuou até mesmo com informações do título marcados nos cilindros moldados. A gravação era direta e a duplicação mecânica (pantográfica) com velocidade de 120, 144 ou 160rpm; com 100tpi 17.

\section{Edison Gold-Moulded Record (1902-1912)}

Os cilindros moldados, figura 7 , têm a seguinte composição química: carbonato de sódio 12,9\%; hidróxido de sódio $0,6 \%$; alumínio $0,2 \%$; ácido esteárico $60,1 \%$; carnaúba $12,8 \%$; negro-de-fumo $12,8 \%$ e cerasina $0,5 \%$ [21]. A velocidade de gravação foi padronizada em 160rpm; com $100 t p i$ [17].

\section{Edison Amberol Record(1908-1912)}

A composição química dos cilindros amberol é: ácido esteárico $31,6 \%$; ebonite $13,2 \%$; cerasina $2,3 \%$; sal soda $7,1 \%$; soda cáustica $33,0 \%$ alumínio $12,8 \%$ [21]. A velocidade de gravação padronizada foi mantida em 160rpm; $200 t p i$ [17.

\section{Edison Blue Amberol Record(1912-1929)}

Composição química: álcool desnaturado $53 \%$, água $35,7 \%$, acetona $8,9 \%$, azul de metila BB 1,8\% [17. A velocidade de gravação padronizada foi mantida em 160rpm; 200tpi 17.

\subsection{Construção de um arqueofone}

Para construir um arqueofone, utilizamos um Arduino Uno com ponte H (figura 8); Motor de passo (Dynasyn, 4SQ-120BA 40S); Cápsula fonocaptora magnetodinâmica (AXXIS I, LeSon); Tacômetro digital (CF5135B); Interface de áudio (Presonus, Audiobox 1616VSL); Computa-

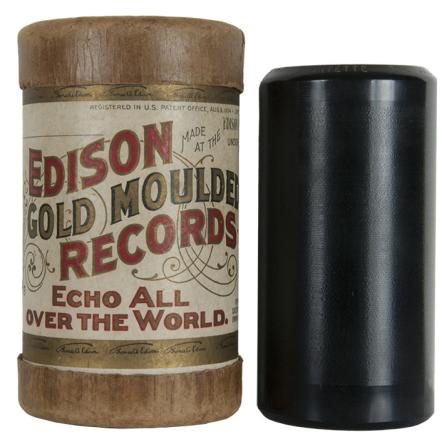

Figura 7: O cilindro tipo Edison Gold-Moulded Record, a primeira media que teve produção em massa por processo de moldagem [17].
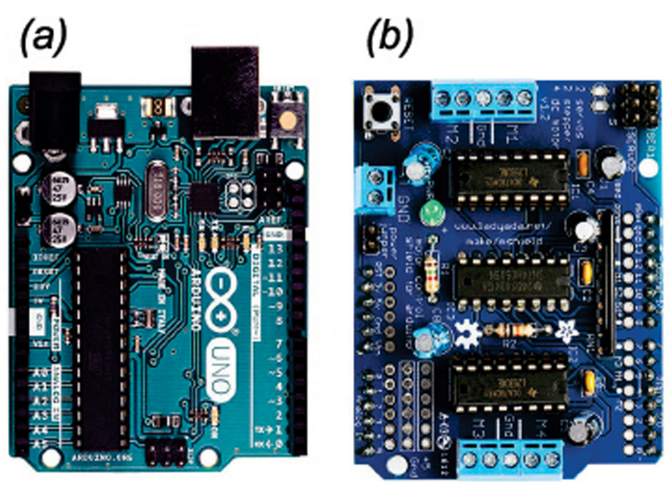

Figura 8: (a) Arduino Uno; (b) Arduino Motor Shild.

dor com software de gravação/edição e uma mesa óptica (Optron, MO-400x600);

A cápsula fonocaptora, é uma cápsula magnetodinâmica com montagem padrão e massa de $4,0 g$. O cantilever da agulha é uma haste de titânio, a agulha é de diamante com ponta cônica com força de trilhagem de 3,0 a $5,0 \mathrm{~g}$. A resposta em frequência é de $20 \mathrm{~Hz}-20 \mathrm{kHz}$ $( \pm 2 d B)$, com sensibilidade de saída de $1 \mathrm{kHz}$ a $5 \mathrm{~cm} / \mathrm{s}$, $50 \mathrm{mV}$; Separação de canais: $18,0 d B$; Balanço entre canais: $2,0 d B$. Os cilindros devem ser reproduzidos com a mesma cápsula, mas com uma agulha mais grossa (safira) para cilindros de dois minutos. A velocidade de gravação do cilindro pode variar e, portanto, as velocidades de reprodução podem não ser exatamente precisas.

\subsection{Transcrição e identificação}

A interface de áudio USB (PreSonus, Audiobox 1616VSL usa conversores de 24bits e taxa de amostragem de até $96 \mathrm{kHz}$ juntamente com uma estação de trabalho de áudio digital $(D A W)$, um sequenciador que tem a finalidade de gravar, editar e tocar áudio digital, para transferir o som do cilindro para o formato padrão .wav. Os sons transcritos devem ser identificados em catálogos 27-31] e tratados antes de serem disponibilizados em meio digital.

\subsubsection{Copyright}

As transcrições são para fins didádicos e, segundo a $U C S B$ Cylinder Audio Arquive [31], todos os registros do selo Edison Records tornaram-se de domínio público, a partir do momento que os bens da gravadora foram transferidos para uma agência federal, a National Park Service.

\section{Resultados}

\subsection{Arqueofone}

A base do arqueofone foi montada sobre uma placa de alumínio anodizado de dimensões 600 x $800 \mathrm{~mm}, 9,5 \mathrm{~mm}$ de espessura e com uma grade de roscas M6 espaçadas em $50 \mathrm{~mm}$ que permite fixar posicionadores e suportes para o motor de passo e o mandril (ver figura 9). O motor de 


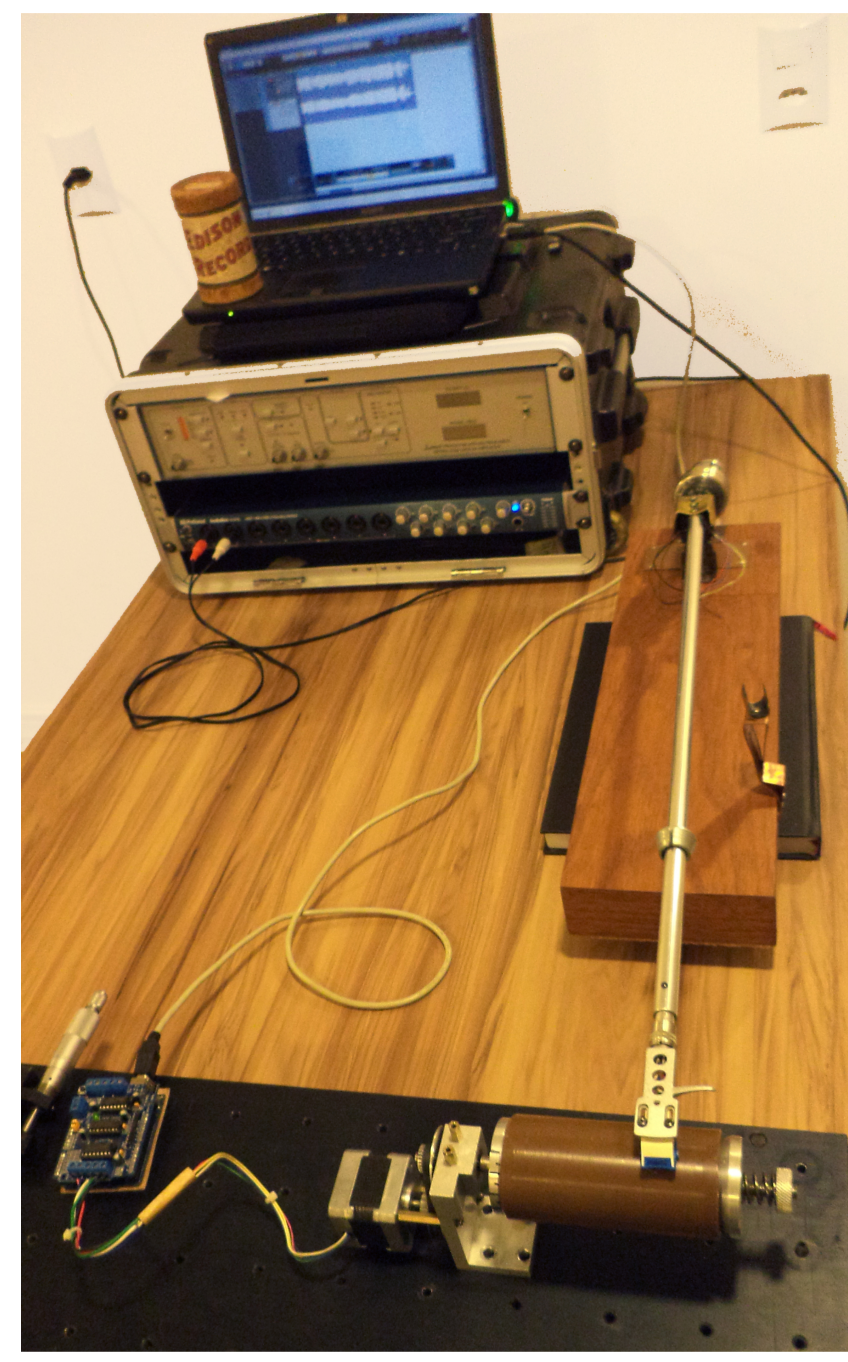

Figura 9: Foto do arqueofone construído no momento da transcrição de um cilindro de cera marrom.

passo com engrenagens acopladas a um mandril regulável, é controlado por computador via Arduino.

Um contador de giros com um mostrador digital indica a rotação do cilindro em rpm. A cápsula fonocaptora está acoplada a um braço de $60 \mathrm{~cm}$. A base do braço é giratória e tem um contrapeso para balancear a trilhagem da agulha no sulco do cilindro. O sinal elétrico captado pela cápsula é enviado a um pré-amplificador com conexão USB para transferir o som registrado no cilindro em formato digital no computador.

\subsection{Transcrição e identificação}

Os registros fonográficos dos cilindros foram digitalizados no formato .wav, PCM, 32bits, a uma taxa de 44,1 $\mathrm{kHz}$. Os cilindros Edison Golden Moulded Records, Edison Amberol Records e Edison Blue Amberol Records foram facilmente identificados por marcações escritas no próprio cilindro e confirmados em catálogos. Os cilindros de cera marrom Edison Records devem ser tocados para verificar o conteúdo, eles têm um anúncio falado no início da gravação que geralmente inclui o título da seleção, o nome do artista e o nome da empresa de gravação. No caso dos cilindros de cera marrom, é difícil saber se o registro transcrito é de uma matriz original ou uma cópia. Os sons digitalizados neste trabalho foram tratados e estão disponíveis no sitio: www.edisonrecords.bandcamp.com

\section{Discussão}

O Arduino é uma plataforma que foi construída para promover a interação física entre o ambiente e o computador utilizando dispositivos eletrônicos de forma simples e baseada em softwares e hardwares livres 32. A utilização do Arduino, da interface de áudio e do computador, serviram como agentes transformadores no processo de ensino-aprendizagem dos princípios físicos envolvidos nas técnicas mecânicas de gravação/reprodução de som, ao diminuir a distância histórica entre o estudante e a tecnologia da época. O código Arduino utilizado para controlar a velocidade de rotação do arqueofone é apresentado no Apêndice.

Todos os cilindros foram transcritos em uma única velocidade de 60rpm. Depois, os áudios tiveram a velocidade alterada para aproximar da velocidade original de rotação de gravação. A transcrição é ligeiramente melhor quando é obtida do cilindro do lado contrário do lado convencional e a agulha lê do fim para o início. A manipulação de áudio digital permite chegar próximo do registro original. A transcrição dos cilindros de cera de 2 minutos é delicada, ocorre desgaste da cera enquanto que os de quatro minutos, principalmente os Edison Blue Amberol, sofrem menos desgates.

Os cilindros de cera moldados, representam o marco inicial da indústria fonográfica, transformaram o som intangível da música em objetos materiais que poderiam ser vendidos e predominaram entre os consumidores por meio século. $\mathrm{O}$ suporte físico de registro fonográfico teve uma evolução passando dos cilindros de cera para os cilindros de celulose, mas a técnica de gravação por corte vertical permaneceu a mesma. Entretanto, um fonógrafo original construído para reproduzir um dos formatos não pode ser utilizado para reproduzir outro formato.

\section{Conclusão}

Estudamos a técnica de gravação mecânica conhecida como Hill and Dale a partir da construção de um aparato mecânico simples, um arqueofone. O arqueofone construído utiliza uma cápsula fonocaptora na extremidade de um braço longo em base giratória e uma interface de áudio conectada a um computador, para transcrever o som centenário registrado em cilindros de cera ou de celulóide. Os sons digitalizados foram identificados em catálogos e estão disponíveis na web. O arqueofone tem grande utilidade em sala de aula para discutir fatos históricos da indústria fonográfica, conceitos de acústica, transdução acústica-mecânica e mecânica-elétrica, além 
de ser uma importante ferramenta de pesquisa em etnomusicologia.

\section{Agradecimentos}

Aos alunos da disciplina EAC1028 - Técnicas de Gravação, do curso de Engenharia Acústica, do Centro de Tecnologia da Universidade Federal de Santa Maria.

\section{Material suplementar}

O seguinte material suplementar está disponível online: Apêndice - Código Arduíno

\section{Referências}

[1] A. McNeese, J.D. Sagers, D. Jason, R.D. Lenhart, P.S. Wilson and S. Preston, Proc. Meet. Acoust. (ASA161, Seattle, 2011), v. 12, p. 1-32.

[2] G. Brock-Nannestad and J.M. Fontaine, J. Acoust. Soc. Am. 123, 5 (2008).

[3] R. Cowen, Science 335, 3066 (2012).

[4] T.A. Edison, US Patent 200,521 (1878).

[5] T.B Lambert, US Patent 664,223 (1900).

[6] M.C. Lefferts, US Patent 672,909 (1901).

[7] T.O. Pinto, Cem Anos de Etnomusicologia e a Era Fonográfica da Disciplina no Brasil (Contexto, CNPq, 2004).

[8] Laboratório de Pesquisas em Etnicidade, Cultura e Desenvolvimento, Rondônia 1912. Gravaçôes Históricas de Roquette-Pinto (Museu Nacional, Rio de Janeiro, 2008).

[9] E. Berliner, US Patent, 534-543 (1895).

[10] H. Chamoux, Revue du Musée des arts et métiers 27, 25 (1999).

[11] http://www.christerhamp.se/phono/ acesso em 05/10/2017.

[12] E. Berliner, US Patent, 372-786 (1887).

[13] G.L. Frow and A.F. Sefl, The Edison Cylinder Phonographs: A Detailed Account of the Entertainment Models Until 1929 (GL Frow, Kent, 1978).

[14] A.G. Webster, PNAS 5, 7 (1919).

[15] P.A. Martin, J. Acoust. Soc. Am. 116, 3 (2004).

[16] G.L. Hogan, US Patent 673,396 (1901).

[17] S. Brylawski, M. Lerman, R. Pike and K. Smith, ARSC Guide to Audio Preservation (Association for Recorded Sound Collections, Pittsburgh, 2015).

[18] T.A. Edison, US Patent 400,646 (1889).

[19] http://www.amplitudemodulation.com.au/edison. html acesso em 05/10/2017.

[20] H.M. Franceschi, Registro Sonoro por Meios Mecânicos no Brasil (Studio HMF, Rio de Janeiro, 1984).

[21] W.R. Raymond, ARSC Journal 26, 2 (1995).

[22] T.B. Lambert, US Patent 645,920, (1900).

[23] T. Nakamura, T. Ushizaka, J. Uozumi and T. Asakura, Proc. SPIE 3190, 304 (1997).

[24] V. Fadeyev and C. Haber, JAES 51, 1172 (2003).

[25] E. Ahrens, E. Richter and H.R. Kuhn, US Patent 3,077,521 (1963).

[26] A.R. Groh, Audio Engineering Society Convention 55 (AES55, Los Angeles, 1976), p. 385-390.
[27] A. Sutton, Edison Two-Minute and Concert Cylinders American Series, 1897-1912 (Mainspring Press, Littleton, 2015), p. 1-398.

[28] http://sounds.bl.uk/related-content/TEXTS/029IEDIGX1907XXX-0000A0.pdf, acesso em 05/10/2017.

[29] A. Sutton, Edison Amberol Cylinders: Complete American and Foreign Issues, 1908-1913 (Mainspring Press, Littleton, 2013), p. 1-252.

[30] A. Sutton, Edison Blue Amberol Cylinders: Complete American, Foreign, and Special-Use Issues, 1912-1929 (Mainspring Press, Littleton, 2005), p. 1-448.

[31] http://cylinders.library.ucsb.edu/, acesso em $05 / 10 / 2017$.

[32] M.A. Cavalcante, C.R.C. Tavolaro e E. Molisani, Rev. Bras. Ens. Fis. 33, 4503 (2011). 